\title{
Autonomous UAV Navigation Using Reinforcement Learning
}

\author{
Mudassar Liaq and Yungcheol Byun
}

\begin{abstract}
Over the last few years, UAV applications have grown immensely from delivery services to military use. Major goal of UAV applications is to be able to operate and implement various tasks without any human aid. To best of our knowledge, in the existing works for autonomous navigation for UAV's, ideal environments (e.g., 2D) are considered instead of realistic or special hardware are used (e.g., nine range sensors) to navigate through an ideal environment. Therefore, in this thesis, we aim to overcome the limitations of the existing works by proposing a model for navigating a drone in an unknown environment without any human help or aid. The goal of this research is to navigate from location A to location B in unknown terrain without having any prior knowledge about the terrain using default drone sensors only. We present a model which is compatible with almost every off-the-shelf drone available in the market. Our methodology utilizes only standard drone sensors which are attached to almost every drone. These include a camera, GPS, IMU, magnetometer, and barometer. Our methodology uses 3D, POMDP, and continuous environment. It also takes into account environmental factors such as winds and rain. Our main contributions are: 1) We are using realistic environment model including factors like rain and wind. 2) We are only using onboard computing resources to run our model instead of some external server. 3) We were able to fly the achieve complete autonomous flight using only standard drone sensors.
\end{abstract}

Index Terms-Unmanned arial vehicle, global positioning system, inertial measurement unit, partially observable Makarovian decision process.

\section{INTRODUCTION}

With the rapid advancements in the technology, the fields of robotics and mechatronics have drastically upgraded in terms of their role in the modern business and different industries. UAV also known as a drone is an aircraft that flies without any human pilot and are controlled remotely.

For a quadcopter to perform in an indoor environment, it requires to operate with agility and efficient feedback without losing control. Stabilization is needed for an outdoor environment for a drone to operate against external forces. In short, for both situations, a stable flight is immensely important for a drone to function effectively. This can help a quadcopter to survive in extreme external factors e.g., heavy rain or wind as it will be able to prevent crashing. In order to use these applications for navigation and stabilization, UAVs and drones need to be supplied with a series of sensors e.g., depth cameras, accelerometer, gyroscope, magnetometer, etc.

Manuscript received received August 12, 2019; revised October 15 , 2019.

Mudassar Liaq and Yungcheol Byun are with the Department of Computer Engineering, Jeju National University, Jeju, South Korea (email: mudassar192@hotmail.com, ycb@jejunu.ac.kr).

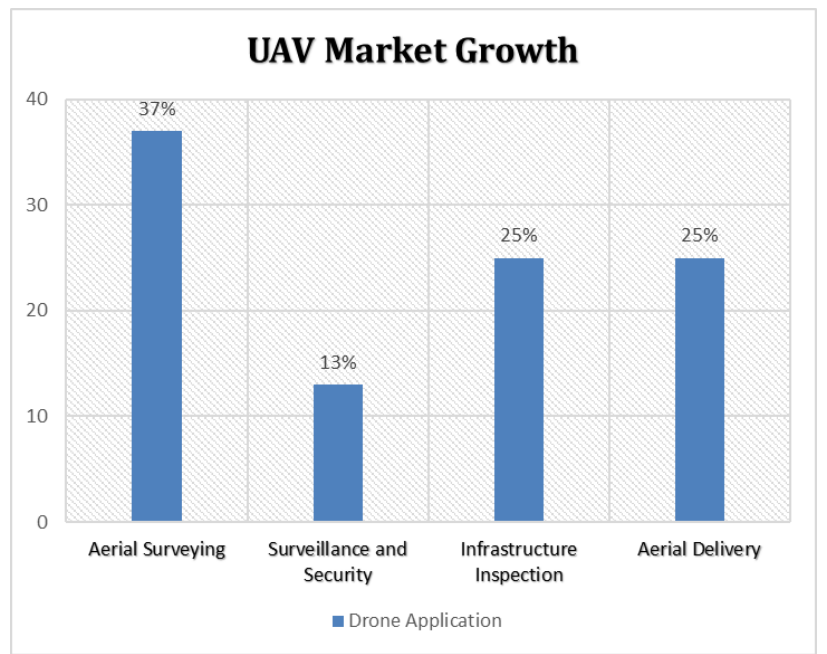

Fig. 1. UAV applications (Market Growth).

Apart from this, applications of drones can be observed in many fields such as accident reporting, infrastructure inspection, crop monitoring, etc. Fig. 1 is from a survey which presents the growth rate of different UAV applications, it can be seen that aerial surveying and mapping is growing at a higher rate (Murfin) [1].

Quadcopters are a more specific form of an aerial vehicle known as multicopter having an arbitrary number of rotors. Quadcopters are driven by four rotors. In the 1920s, when quadcopters were first introduced, they were unable to gain popularity due to challenges like having comparatively large size and weight, mechanical complexity, control management, and etc. [1], [2].

Data in large volumes are often spawned by drones and UAVs. These UAV applications will only be useful for the consumer if this data is managed efficiently and effectively without requiring extra efforts. Therefore, AI (Artificial Intelligence) seems to cover these challenges as nowadays every other company or industry is using AI techniques, machine learning or deep learning to process immense amount of data. There are many AI based tasks that deal with image recognition, UAVs are also required to perceive and understand the environment, detect and avoid collisions in order to achieve a smooth flight and motion planning is also another task which requires $\mathrm{AI}$ and machine learning to play an active part as shown in Fig. 2.

Autonomous navigation in unknown terrain is an exponentially hard task due to unavailability of preconstructed maps or path planning. In this thesis, a deep RL based framework is developed for UAVs navigation in unknown large-scale complex environments.

Specifically, first, an efficient policy based DQN is designed, which comprises of convolutional and fully connected layers to extract important features from images taken through depth vision camera. These features are then 
combined with inputs taken directly from other sensors, e.g., IMU or magnetometer, etc. and are passed to a fully connected layer. This is followed by a policy-based Qlearning approach to apprehend UAV navigation.

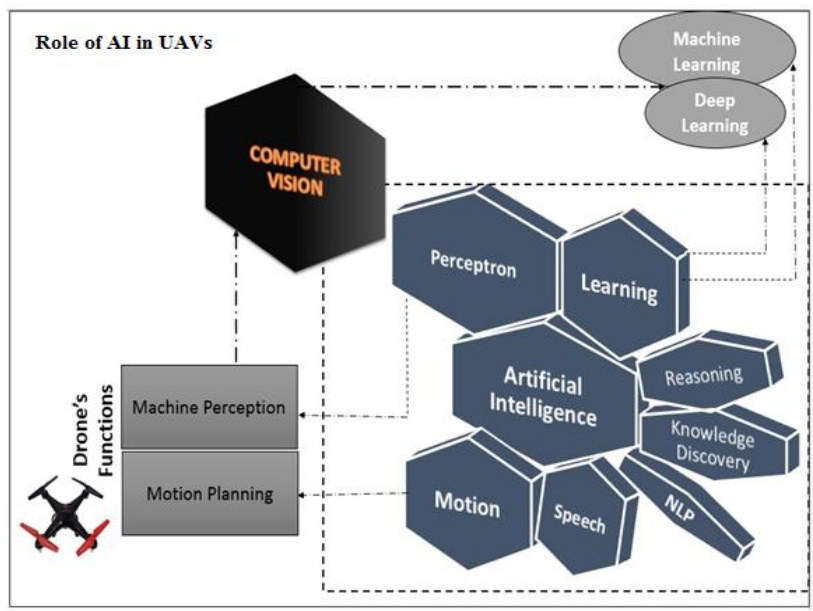

Fig. 2. Role of AI in UAVs.

\section{RELATED WORK}

Relatively much work has been going on with respect to automatic UAV navigation with machine learning algorithms but less or no work has been done on unknown path travel by UAV. Su Yeon Choi and Dowan Cha in their paper "Unmanned aerial vehicles using machine learning for autonomous flight; state-of-the-art" has divided their research areas into three categories parameter tuning, adaptive control and real-time path planning [3].

Smolyanskiy, et al. "Toward low-flying autonomous MAV trail navigation using DNN for environmental awareness" proposes a micro air vehicle system to follow trails in an unstructured outdoor environment. Drawbacks are NVidia custom hardware chips (TX1+J120) are used and not fully autonomous (Human intervention required) [4]. For non-colliding paths for multi-agent UAV's cooperative planners have been developed. It has inaccuracies. Geramifard et al. studies on the ICCA for combining cooperative planners and RL techniques as a framework [5][9]. Zhang et al. [10] propose a CGLA, which is made for path planning on multiple UAV cooperation [11]. In CGLA, to balance between the economy of paths and collision avoidance, the parameters are trained. The individual weight matrix and cost matrix are proposed for an efficient path planning of both single and multiple UAVs [3]. Based on the geometric distance and risk information shared among UAVs, the individual weight matrix is calculated and adaptively updated. The optimal path from a starting point to a target point is calculated. The simulation results validate the effectiveness and feasibility of CGLA for safe navigation of multiple UAVs. However, it is required to utilize the coarse and fine grid to design a fast path planning algorithm in the proposed framework and apply the algorithm to an actual UAV [3].

There are results to avoid a collision for UAV using laser range finders or Kinect cameras. Vandapel et al. [12] avoid a collision in 3D space using lidars, and Bachrach et al [13], and Bry et al. [14] depict obstacle avoidance in an unknown room using scanning lidars for SLAM [12-[15]. Bachrach et al. use Microsoft Kinetic camera in an unknown room [15]. However, these research works are required to improve on problems of power supply, payload, UAVs cost, and the reliability of localization. As a result, Achtelik et al. [16], Wendel et al. [17], Fraundorfer et al. [18] created maps by a single camera, or stereo cameras [15], [16], [18]. Achtelik et al. [19] created sparse maps of the environment using a single, cheap camera [15]. Wendel et al. [9] created dense maps of the environment using a camera [8]. Fraundorfer et al. [18] demonstrated accurate depth estimation and localization using stereo cameras. However, even though the algorithms are reasonably fast to avoid a collision, they were still too computationally expensive for UAVs flight. Recently, many researchers have studied path planning and formation light techniques [20]-[23].

The environmental model defines the space in which drone navigates. In [22], the 2D environmental model is utilized. In [21], a simplified model in a 3D environment is used where the impact of drag forces on the drone is ignored. In [22], the 3D model is utilized for modeling. In our work, we have considered a complete 1D environment with consideration to all the environmental factors, which include winds, rain as well as drag effect created by these elements.

Observational Space defines how the environment behaves when some action is performed. In the deterministic environment, if given a state, action pair next state can be guaranteed while in a stochastic environment, it's not guaranteed that state, action pair will generate the same output every time. In [21], [22], observational space is set to deterministic while [4], [24], [25] have used stochastic model. We have also utilized the stochastic observational model for our environment as its closer depiction of realworld scenarios.

Observability refers to how much environmental state is available to UAV at any given point in time. In [4], [22], completely observable environment is utilized. In [4], [21], [25], assumed the partially observable environment is assumed where some part of the state is available at any given time instead of whole (which is in case of fully observable). We have taken environment observability to be partial.

In [14], Model based RL along with random shooter model is deployed. In [22], [25], model free RL is used while in [4], [21], standard DNNs are exploited. In our work, we are utilizing model free RL.

In [24], a separate module for image processing is used, but detailed explanations are not specified in the paper. In [21], [22], [25], no camera module is utilized at all. Therefore, there is no image processing module. In [4], a separate module for video processing is utilized. For this purpose, the YOLO module is utilized. In our work, we are not utilizing any separate module for video processing. The data is instead fed into the $\mathrm{NN}$, which utilizes it for generating action probabilities.

Ideally, a UAV should not rely on any other external help for its decision making. In [24], however, an external ROS server is used as a helping node. In [22], the extra onboard PID module is used. In [4], [21], [25], any helper modules are not required. In our work, we are not utilizing any external helper modules except the NN. 
Drone type is essential as it plays a huge part in deciding the adaptability of research in real-world scenarios. Off the shelf, drones are the desired scenario as it increases the chances of adaptability to very high. In [21], [22], [24], off the shelf drones are used. In [4], [25], customized drones are used for the experiment. In our work, we are using off the shelf drone.

In [24], an extra ROS server is used, which is deployed on a high-end machine. In [21], [22], no off-board resources are deployed in their work. In [4], TX-1 and AuVedia chips are placed on a drone for processing. In [25], an extra nine range sensors are deployed for the experiment. In our work, we have not utilized any off-board computing resource and have used only off the shelf drone.

When a model is pushed into drone memory which is already trained on some existing dataset, then that model is called pre-trained model and process is called pre-training. In [22], [24], [25], they have not used any pre-training. In [21], 512 initial and 1024 branching trajectories are utilized for pre-training the model. In [4] IDSIA dataset is being utilized for pre-training. In our work, we have not utilized any pre-training.

In [4], state action pairs from the previous 15 iterations are used as an input for the NN. In [22], the 3D environmental state is deployed as its primary data source. In [21], orientation, position, and velocity are exploited as input. In [4], cameras are used as input. In [25], gyroscope, GPS, and range sensors are deployed as input. In our work, we have utilized the camera, IMU, GPS, barometer, and magnetometer as input

\section{PROPOSED METhodology}

The baseline idea is to autonomously navigate a drone in an unknown environment, which means in whatever environment a drone is, it must be able to navigate effectively and efficiently. This is achieved by using policy based DQN, a deep learning technique. There are five major modules in our system named as Input, Output, System, Environment, and Reward. Input deals with the sensor generated values. The reward is calculated as an indicator of an action's feedback. The more the action is performed accurately, the higher is the reward. Next, this reward is forwarded to the system, where our policy-based DQN model (described in next section) processes all the inputs and generates output in the form of the probability distribution of actions. The most favorable action is performed by the quadcopter; action feedback is recorded, passed to our DNN, and the cycle continues. The environment here represents the terrain where our quadcopter is flying or navigating. Environment generates many parameters like sensor's readings and some states on which the reward function is based.

We propose a model for navigating a drone in an unknown environment without any human help or aid. The goal is to navigate from location A to location B in unknown terrain without having any prior knowledge about the terrain using default drone sensors only. This work aims to address the limitations of existing research works by taking into account the following parameters as primary contributions of this work are following. A continuous domain is being considered rather than a discrete one. The simulation environment is 3D. 3D navigation is enabled, i.e., the quadcopter can navigate across all three-axis $\mathrm{x}, \mathrm{y}$, and $\mathrm{z}$. High model accuracy (High success rate, low stray, and failure rate). The model is completely autonomous. Performance evaluation is done on multiple environment datasets.

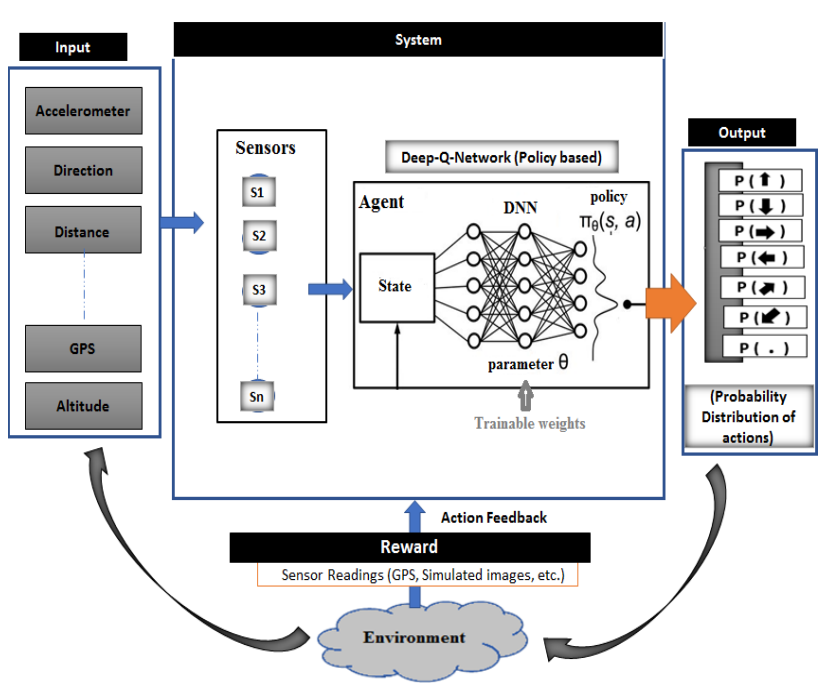

Fig. 3. Proposed system design.

In this work, we are using DQN to predict the policy directly instead of Q-value. The advantage of using policybased approach is it can work for continuous domains. Fig. 3 is the complete system design covering all the inputs, methods, and outputs. The elements of DQN are explained in Table I. An agent is an entity which decides and takes certain actions. State refers to the internal state of our DNN, e.g., learning weights. An action is a decision made by the agent to make quadcopter do some movement, e.g., moving up or down. The reward is a measure of how good an agent is performing.

\begin{tabular}{|c|c|c|}
\hline \multicolumn{3}{|c|}{ TABLE I: KEY RL ELEMENTS } \\
\hline Key RL Terms & Explanation & Examples \\
\hline Agent & $\begin{array}{l}\text { Decision-making entity } \\
\text { that takes action }\end{array}$ & DQN \\
\hline State & NN internal weights & Camera, GPS \\
\hline Action & $\begin{array}{l}\text { A decision made by the } \\
\text { agent to take any of the } \\
\text { actions mentioned above }\end{array}$ & $\begin{array}{l}\text { Quadcopter moving } \\
\text { left or right }\end{array}$ \\
\hline Reward & $\begin{array}{l}\text { How good agent is } \\
\text { performing }\end{array}$ & Distance from goal \\
\hline
\end{tabular}

\section{EXPERIMETAL SETUP}

To experiment with deep learning, RL, and computer vision algorithms for UAVs, we have used AirSim as a simulation tool. AirSim enables us to use different APIs to retrieve images, control the vehicle, etc. Table II presents the components and their details for the simulator. AirSim is used as a platform for simulations. It provides different APIs to enable communication with vehicles in the simulation environment. Some example APIs are also given in Table II.

These APIs can be deployed on a computer on the vehicle 
with which you aim to interact, in our case, its quadcopter as these APIs are also accessible as an independent crossplatform library. Code written in the simulator can later be executed on the real UAVs. Table III presents the system specifications. We used python 3.6 as a programming language, Tesorflow version 1.13, Cuda version 10.0, cudann version 7.4.2.

TABLE II: DETAILS OF THE SIMULATION ENVIRONMENT

\begin{tabular}{|c|c|c|}
\hline Components & Name & Details \\
\hline $\begin{array}{c}\text { Simulation } \\
\text { tool/Platform }\end{array}$ & AirSim & $\begin{array}{c}\text { Open-source } \\
\text { simulator for UAVs, } \\
\text { cars and more }\end{array}$ \\
\hline AirSim APIs & $\begin{array}{c}\text { simGetImage, } \\
\text { reset, } \\
\text { confirm connection, } \\
\text { simGetObjectPose, } \\
\text { simGetCollisionInfo }\end{array}$ & $\begin{array}{c}\text { To retrieve images, } \\
\text { get state, control the } \\
\text { vehicle and for many } \\
\text { other tasks }\end{array}$ \\
\hline Vendor & Microsoft & $\begin{array}{c}\text { AirSim currently } \\
\text { supports these } \\
\text { sensors data }\end{array}$ \\
& IMU, & magnetometer, \\
& barometer & \\
\hline
\end{tabular}

TABLE III: SYSTEM SPECIFICATIONS

\begin{tabular}{|c|c|}
\hline \multicolumn{2}{|c|}{ TABLE III: SYSTEM SPECIFICATIONS } \\
\hline Components & Versions \\
\hline Operation System & Linux (x64) \\
\hline Python & 3.6 \\
\hline Tensorflow & 1.13 \\
\hline Cuda & 10.0 \\
\hline CudaNN & 7.4 .2 \\
\hline
\end{tabular}

\section{RESUlts}

The drone is initially spawned in a random location on the map, and the final destination is set to a predefined location (usually midpoint in the map). The goal of the drone is to fly from its random initial position to the final destination without any collisions.

The speed and altitude of the UAV range between 0 to $10 \mathrm{~m} / \mathrm{s}$ and 5 to $200 \mathrm{~m}$ respectively. The episode length (i.e., time step) can be up to 200. The network parameters are learned by using Adam Optimizer with a learning rate of 103. The discount factor $\gamma$ for the experiments is varied in the range of 0.91 to 0.99 .

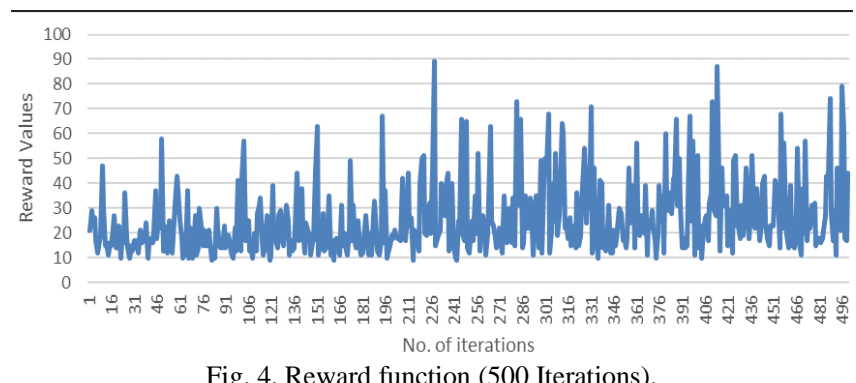

The graph shown in Fig. presents a trend of reward function values when we run the program for 500 epochs. It can be observed that the network was learning very slowly. This is a known behavior as the network is experimenting with different outputs to check which strategy works. After a few hundred iterations, it starts to learn about some successful strategies, and reward gradually starts to build up.

The only measurable factor that can quantify that the
DQN algorithm is working properly is a reward. In our scenario, the reward function comprises of the combination of hubber loss and distance from the destination.

The graph shown in Fig. 2 shows a trend of reward function values when we run the program for 1000 epochs. After a few small tweaks and an increasing number of iterations to 1000 , we can observe that the reward is maxing out. The reward was capped at 200. As the iterations increase, the reward values show a rapid increase, respectively.

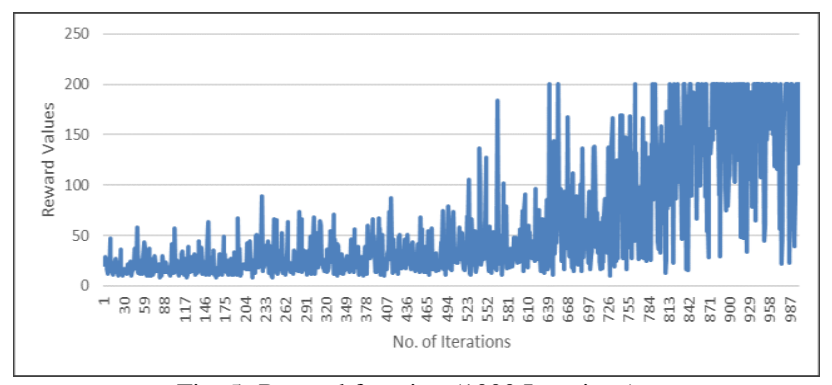

Fig. 5. Reward function (1000 Iterations).

In any navigation, the most important part is a realistic and complete environmental model. It's a baseline for interaction as a strong environmental model would result in strong results. In [21], [22], complete environments are used. In [22], a 2D environment is used, which in itself reduces the complexity model. It also removes a lot of complication from the problem statement. In [21], the 3D model is used, but a very simplified model is assumed. All the drag forces that act on the drone and make navigation a difficult task are ignored. In [24], [4], [25], completely realistic environment is utilized. In our work, we are also utilizing a completely realistic environment taking into account all the factors that make the environment more stochastic.

Model free RL is not the golden standard for navigation tasks. Its performance is best among all the available methods. In [23], [24], model based RL is utilized. In [4], simple DNNs are deployed. In [5], [21], [22] Deep model free RL is used.

It's very important that what kind of hardware is utilized for the navigation as it has a direct impact on its adaptability if someone is getting very good results but is utilizing very specific hardware, then its less useful as compared to one which has relatively less impressive results but is using commodity hardware. [21], [22], [24] are using commodity hardware, so their work is extensible. In [4], very specific Nvidia TX1, AuVedia 120, and three cameras are utilized. In [25], an extra nine range sensors were attached to their UAV. In our work, we are using off the shelf drone with all the standard sensors. This makes our work more relevant for implementation by industry.

This refers to the ability to learn from its mistakes as its flies instead of training model explicitly. In [21], [22], [24], [25], deep RL is utilized; therefore, the ability to have a model that learns on the fly exists. In [4], DNN is used; therefore, they lack this ability. We are also utilizing learning on the fly ability as we are also using model free RL.

Onboard processing is an important benchmark in drone navigation. Nowadays, it's necessary that the drone should 
have all the processing onboard to guarantee complete autonomy.

TABLE IV: PERFORMANCE EVALUATION SUMMARY

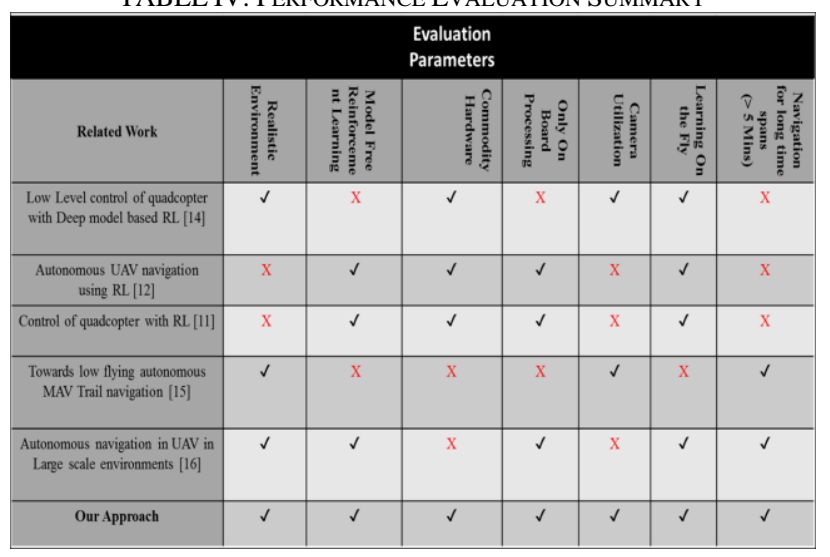

This parameter defines whether the authors were able to fly drone autonomously for more than 5 mins. In [4], they were able to achieve 6 seconds flight from 3 minutes of data If they tweaked with the training data, their results deteriorated. In [22], results were presented for the 2D environment. Therefore, the work was only good enough for the testbed. For an outdoor, real-world 3D environment where drag forces effect, the model couldn't perform well. In [21], drone stabilization was achieved but not the navigation. Their work shows that the drone can hover for more than 5 minutes but cannot navigate through the environment. Another issue in this work [21] was a simplified model assumption which makes even hovering unstable in a real-world environment.

In [24], an extra ROS server is deployed. In [21], [22], [25], only using onboard processing power is exploited. In [4], extra chips beside the traditional hardware are utilized. TX-1 and Auvedia-120 chips are being used. In our work, we are also not utilizing any off-board processing.

All of these factors are summarized in Table IV. We have concluded from the above considerations that most of the works only cover a few of the above considerations. This makes our work stand out from rest as no one has covered all these factors in their research (till date) along with our state-of-the-art results to the best of our knowledge. The work that was closest to our implementation is presented in [25], but it also misses a few key aspects (e.g., customized hardware, extra sensors, etc.). We were also able to achieve state-of-the-art success rate in our work. In our research work, we have taken into account all these factors and have completed autonomous navigation.

\section{CONCLUSION}

We have summarized the critical factors that are important in drone navigation scenario. We have concluded from the above considerations that most of the works only cover a few of the above considerations. This makes our work stand out from rest as no one has covered all these factors in their research (till date) along with our state-ofthe-art results to the best of our knowledge. The work that was closest to our implementation is presented in [5], but it also misses a few key aspects (e.g., customized hardware, extra sensors, etc.). We were also able to achieve state-ofthe-art success rate in our work. In our research work, we have taken into account all these factors and have completed autonomous navigation.

\section{ACKNOWLEDGMENT}

This research was supported by the 2018 scientific promotion program funded by Jeju National University.

\section{REFERENCES}

[1] Money Talks: Drone Investment Trends Update. [Online]. Available: https://www.droneii.com/drone-investment-trends-update

[2] UAV Report: Growth trends \& opportunities for 2019. [Online]. Available: https://www.gpsworld.com/uav-report-growth-trendsopportunities-for-2019/

[3] S. Y. Choi and D. Cha, "Unmanned aerial vehicles using machine learning for autonomous flight; state-of-the-art," Advanced Robotics, 2019.

[4] Smolyanskiy et al., "Toward low-flying autonomous MAV trail navigation using deep neural networks for environmental awareness," IROS, 2017.

[5] J. Redding, A. Geramifard, A. Undurti et al., "An intelligent cooperative control architecture," in Proc. American Control Conference, 2010, pp. 57-62.

[6] J. Redding and A. Geramifard, "How JP. actor-critic policy learning in cooperative planning," in Proc. AAAI Spring Symposium: Embedded Reasoning, 2010.

[7] A. Geramifard, J. Redding, N. Roy et al., "UAV cooperative control with stochastic risk models," in Proc. American Control Conference (ACC), 2011, pp. 3393-3398.

[8] A. Geramifard and J. Redding, "How JP. intelligent cooperative control architecture: A framework for performance improvement using safe learning," J Intel Robot Syst. vol. 72, no. 1, pp. 83-103, 2013 .

[9] B. Zhang, W. Liu, Z. Mao, et al., "Cooperative and geometric learning algorithm (CGLA) for path planning of UAVs with limited information," Automatica, vol. 50, no. 3, 2014.

[10] H. Xu and L. Carrillo, "Fast reinforcement learning based distributed optimal flocking control and network codesign for uncertain networked multi-UAV system," Unmanned Systems Technology XIX; International Society for Optics and Photonics, vol. 10195, p. $1019511,2017$.

[11] N. Vandapel, J. Kuffner, and O. Amidi, "Planning 3-d path networks in unstructured environments," in Proc. the 2005 IEEE International Conference on Robotics and Automation, 2005, pp. 4624-4629.

[12] A. Bachrach, R. He, and N. Roy, "Autonomous flight in unknown indoor environments," Int J Micro Air Vehicle. vol. 1, no. 4, pp. 217228,2009

[13] A. Bry, A. Bachrach, and N. Roy, "State estimation for aggressive flight in GPS-denied environments using onboard sensing," in Proc. 2012 IEEE International Conference on Robotics and Automation, 2012, pp. 1-8.

[14] A. Bachrach, S. Prentice, R. He et al., "Estimation, planning, and mapping for autonomous flight using an RGBD camera in GPSdenied environments," Int J Rob Res. vol. 31, no. 11, pp. 1320-1343, 2012.

[15] M. Achtelik, M. Achtelik, S. Weiss, and R. Siegwart, "Onboard IMU and monocular vision based control for MAVs in unknown in-and outdoor environments," in Proc. 2011 IEEE International Conference on Robotics and Automation, pp. 3056-3063.

[16] A. Wendel, M. Maurer, G. Graber, et al., "Dense reconstruction onthe-fly," in Proc. 2012 IEEE Conference on Computer Vision and Pattern Recognition, 2012, pp. 1450-1457.

[17] F. Fraundorfer, L. Heng, D. Honegger et al., "Vision-based autonomous mapping and exploration using a quadrotormav," in Proc. 2012IEEE/RSJ International Conference on Intelligent Robots and Systems, 2012, pp. 4557-4564.

[18] Q. Luo and H. Duan, "Distributed uav flocking control based on homing pigeon hierarchical strategies," Aerosp Sci Technol., vol. 70, pp. 257-264, 2017.

[19] D. Lee, S. Kim, and J. Suk, "Formation flight of unmanned aerial vehicles using track guidance," Aerosp Sci Technol., vol. 76, pp. 412420, 2018.

[20] L. Ambroziak and Z. Gosiewski, "Two stage switching control for autonomous formation flight of unmanned aerial vehicles," Aerosp Sci Technol., vol. 46, pp. 221-226, 2015. 
[21] H. Jemin et al., "Control of a quadrotor with reinforcement learning," IEEE Robotics and Automation Letters, vol. 2, no. 4, pp. 2096-2103, 2017.

[22] P. Huy et al., "Autonomous uav navigation using reinforcement learning," arXiv preprint arXiv: 1801.05086

[23] K. William et al., "Reinforcement learning for UAV attitude control," ACM Transactions on Cyber-Physical Systems, vol. 3, no. 2, p. 22, 2019.

[24] L. Nathan et al., "Low level control of a quadrotor with deep modelbased reinforcement learning," arXiv preprint arXiv:1901.03737 (2019).

[25] W. Chao et al., "Autonomous navigation of UAVs in large-scale complex environments: A deep reinforcement learning approach," IEEE Transactions on Vehicular Technology.

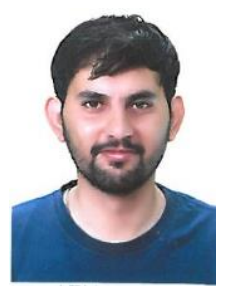

Mudassar Liaq has done B.S from National University of Computer and Emerging Sciences, (NUCES), Islamabad in Computer Science, Pakistan in 2014. For next 3 years, he worked in industry before starting M.S from Jeju National University in September 2017. Currently he is MS student in Jeju National University (JNU). He is also working as a research assistant in ML lab in JNU.

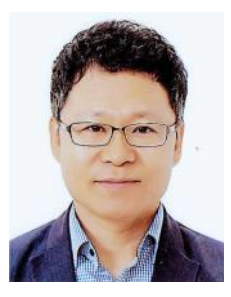

Yung Cheol Byun received his B.S. from Jeju National University, Korea in 1993, M.S and Ph.D. degrees from Yonsei University in 1995 and 2001. $\mathrm{He}$ worked as a special lecturer in SAMSUNG electronics in 2000 and 2001. From 2001 to 2003, he was a senior researcher of Electronics and Telecommunications Research Institute and he promoted to join Jeju National University as an assistant professor in 2003. He is currently a professor of the Department of Computer Engineering, Jeju National University. From 2012 to 2014, he had research activities at University of Florida as a visiting professor. His research interests include pattern recognition \& image processing, artificial intelligence \& machine learning, security based on pattern recognition, home network and ubiquitous computing, u-healthcare and RFID \& IoT middleware system. 